\title{
A clinico-ethical framework for multidisciplinary review of medication in nursing homes
}

Wasim Baqir, Steven Barrett, Nisha Desai, Richard Copeland, Julian Hughes

Northumbria Healthcare NHS Foundation Trust

\begin{abstract}
Residents in care homes are more likely to be prescribed multiple medicines yet often have little involvement in these prescribing decisions. Reviewing and stopping inappropriate medicines is not currently adopted across the health economy. This Health Foundation funded Shine project developed a pragmatic approach to optimising medicines in care homes while involving all residents in decision making.
\end{abstract}

The pharmacist undertook a detailed medication review using primary care records. The results were discussed at a multidisciplinary team (MDT) meeting involving the care home nurse and the resident's general practitioner (GP), with input from the local psychiatry of old age service (POAS) where appropriate.

Suggestions for medicines which should be stopped, changed or started, and other interventions (eg monitoring) were discussed with the resident and/or their family.

Over 12 months 422 residents were reviewed, and 1346 interventions were made in $91 \%$ of residents reviewed with 15 different types of interventions. The most common intervention (52.3\%) was to stop medicines; 704 medicines stopped in 298 residents (70.6\%). On average, 1.7 medicines were stopped for every resident reviewed (range zero to nine medicines; $S D=1.7$ ), with a $17.4 \%$ reduction in medicines prescribed (3602 medicines prescribed before and 2975 after review). The main reasons for stopping medicines were: no current indication (401 medicines; 57\%), resident not wanting medicine after risks and benefits were explained (120 medicines; $17 \%)$, and safety concerns (42 medicines; $6 \%)$.

The net annualised savings against the medicines budget were $£ 77,703$ or $£ 184$ per person reviewed. The cost of delivering the intervention was $£ 32,670$ (pharmacist, GP, POAS consultant, and care home nurse time) for 422 residents; for every £1 invested, £2.38 could be released from the medicines budget.

This project demonstrated that a multidisciplinary medication review with a pharmacist, doctor, and care home nurse can safely reduce inappropriate medication in elderly care home residents.

\section{Problem}

A pilot project involving a pharmacist and general practitioner (GP) in medication reviews was undertaken for 37 residents in a local care home, taking into consideration current co-morbidities, prognosis and resident/family/carer views. The reviews highlighted that many residents (over two thirds) were taking medicines that had no current indication or the medication had no purpose given the co-morbidity. In total, 114 medicines were stopped. A specific case highlighted issues around lack of review and appropriate prescribing.

An 85 year old lady in a care home, who was bed bound and unable to communicate or make decisions, was prescribed 11 regular medicines including medicines to prevent fractures and cardiovascular disease, as well as antidepressants, hypnotics, and antipsychotics for agitation. After discussion with family, preventative medicines were stopped; her agitation resolved and psychotropic medicines were gradually withdrawn with no adverse events.
Clear guidelines exist for starting medicines but there is less guidance for stopping medicines. Prescribers face ethical, legal, and professional challenges when deprescribing. Furthermore, patients are given little opportunity to be involved in decisions about medicines.

\section{Background}

Many of the medicines prescribed to older patients are inappropriate and potentially harmful.[1,2] Care home residents are at a high risk from medicines-related harm as they are older and frailer than the general population, usually take a large number of medicines, are more susceptible to adverse events and often have little say in which medicines are prescribed or administered. The Care Home Use of Medicines Study (CHUMS) demonstrated that two thirds of care home residents were exposed to at least one error with an $8-10 \%$ chance of an error in the processes of prescribing, dispensing or administration of medicines.[3] A report into medicines management in care homes by Age UK and the Health Foundation identified many deficiencies including excess 
and often inappropriate prescribing, lack of structured review, long medication rounds, and lack of resident involvement in decisions about medicines.[4] Stopping medicines can be perceived as problematic as there is less published guidance available when compared to starting medicines.

Involving patients in shared decision making (SDM) about medicines may not be routine practice; barriers include time constraints and the perception among clinicians that SDM is not appropriate for their patients. [5] One study in a care home setting showed that all residents interviewed accepted without question doctors' and nurses' control of prescribing and administration.[6] While prescribers often follow evidence-based guidelines, these guidelines do not necessary reflect the preferences of patients and do not seek to involve them.[7] Studies have highlighted clinician perceptions of patients being attached to their usual medicines, and reluctance to interfere with previous decisions, which contributes to inappropriate prescribing.[8]

\section{Baseline measurement}

Prior to the project, an audit of 49 residents was carried out at a care home in North Tyneside using the STOPP (Screening Tool of Older People's potentially inappropriate Prescriptions) criteria[1]; $63 \%$ (31 residents) were taking one or more inappropriate medicines as defined by the STOPP tool. In a second care home, medication reviews were undertaken by a pharmacist, GP and care home nurse team using a pragmatic approach which took into consideration current co-morbidities, prognosis, and patient/family/carer views. Thirty-seven residents were reviewed and 114 medicines were stopped.

The psychiatry of old age service (POAS) based at North Tyneside General Hospital has a dedicated nursing and residential care home team and a behaviour support service (one consultant and six nurses in all) working into 50 care homes in North Tyneside. A local survey of 94 patients out of a possible 445 residents $(21 \%)$ in 11 homes found a number of potential concerns about medicine use:

$-27 \%(n=25)$ were prescribed antipsychotic medication (20 with
dementia diagnoses)

- $55 \%(n=52)$ were prescribed antidepressant medication

- $35.9 \%(n=33)$ were prescribed hypnotic medication (19 were taking regularly)

- 42\% ( $n=39)$ were prescribed $>1$ psychotropic medicine.

Qualitative interviews with care home staff prior to the project starting identified three main themes: lack of review, with some residents never having had a review, long, and complicated medicines rounds and lack of resident and/or family involvement in decisions about medicines.

\section{Design}

Local learning and national research on the poor quality of medicines use in care homes were the drivers for this project. A team of experts was assembled and a project plan with key milestones created. Funding was successfully obtained from the Health Foundation's Shine 2012 programme.

The intervention had three main components: a detailed review of medicines by clinical pharmacists, a multidisciplinary team discussion and resident (and/or family) involvement. During the medication review process, the following questions were considered:

1. Is the medication currently indicated?

2. Is the medication still appropriate when taking into account co-morbidities?

3. What are the resident's (or family/carers') views?

4. Are there medicines missing that the patient should be taking?

The results of the medication review were discussed within a multidisciplinary team (MDT) comprising of professionals including the clinical pharmacist, care home nurse, general medical practitioner, psychiatry of old age consultant, and POAS staff. The final decisions were made jointly with the resident (or their family) where this was possible.

All residents were monitored for adverse events by care home staff following the intervention and followed up by the pharmacist to ensure suspected negative effects were identified and safely managed. The process was iterative, with rapid feedback from each clinic being used to improve the process.

\section{Strategy}

The first four MDT meetings comprised a pharmacist, care home nurse, general medical practitioner, with nursing and/or consultant input from POAS. Through qualitative interviews with the team it became apparent that the psychiatry team was not needed for the majority of planned interventions as they involved medicines for a range of chronic physical conditions. An alternative model for POAS involvement was developed: residents known to the psychiatry team were highlighted to the team, new residents were referred into the team using existing referral mechanisms or email/ telephone advice was sought. This model provided a better use of psychiatry resources.

The team recognised from initial reviews that involving all residents in decisions was a challenge. In the first PDSA (plan, do, study, act) cycle it was identified that many residents lacked capacity for decisions about medicines and many families were difficult to engage. Following further discussion a four level resident involvement framework was developed as follows:

1. Assume capacity and involve resident

2. Where resident lacks such capacity, ask family member to be involved

3. Where the family member is unable to attend contact them via telephone (and Skype call to Australia in one instance) 
or write a letter to them

4. Where the resident has no family or significant friends, seek independent advocates.

This model was tested and accepted by the clinical team. Resident involvement was further modified in later cycles where the residents (and/or family) were invited to join the MDT. It was recognised that this worked for some but not all residents, so two models were adopted: resident (and/or family) were consulted after the MDT or resident (and/or family) attended the MDT.

The first PDSA cycle involved a care home whose residents were all under the care of a single general practice. As more homes were reviewed it became apparent that many were under the care of multiple general practices and were reviewed by GPs in different ways. Over several cycles four models of working with GPs were developed:

- Model 1: GP attended the MDT and decisions were jointly made with the care home nurse and pharmacist

- Model 2: All interventions were discussed with the GP after the pharmacist review and prior to the MDT

- Model 3: All interventions were discussed with the GP following the MDT but prior to resident involvement

- Model 4: No involvement of the GP. Practice agreed the prescribing pharmacist leads the process. Interventions were recorded in the general practice electronic notes and GPs were given a chance to challenge the interventions. No interventions were challenged.

Data showed that model 1 (where the GP was fully involved) was the most efficient way of working. Model 2 was abandoned after one cycle as it was inefficient, with the pharmacist needing to go back to the GP after the MDT on many occasions. Model 3 was less efficient but was accepted where practices wanted to be involved but could not release GPs to attend MDTs. Model 4 was more cost effective (ie better outcomes for less cost) than models 2 and 3 but less efficient than model 1 and allowed another competent health professional [the pharmacist] to manage this group of patients.

\section{Results}

Reviews were undertaken at 12 main care homes (fully reviewed) and eight additional care homes (partially reviewed). The majority $(n=15)$ were mixed nursing and residential homes, with three being residential and two nursing only.

Altogether, 422 residents were reviewed within 16 general practices. In 382 residents (90.5\%) there were 1346 interventions, with only $9.5 \%$ of residents not requiring any intervention.

The most common intervention was to stop medicines (704 medicines) in 298 residents (70.6\%). On average 1.7 medicines were stopped for every resident reviewed (range 0 to 9 medicines; SD 1.7). This was equivalent to a $17.4 \%$ reduction in medicines prescribed. The most common reasons for stopping medicines were no current indication $(57 \%)$ and residents not wanting to take the medicine after risks and benefits were explained (17\%). Forty-one medicines $(6 \%)$ were stopped because of safety concerns.

Following changes to medicines, nine residents $(2.1 \%)$ experienced potential adverse events. All events were reversible and did not result in harm to the patient.

A patient involvement framework was developed so that all residents could be involved in decisions. Of the 382 residents needing an intervention, 352 were asked to be involved in decisions; 30 residents received interventions which did not require a shared decision about medicine management. Fifty-seven residents (16\%) were fully involved in decisions about medicines. Families were involved for 137 residents (39\%) and letters were sent to families of $141(40 \%)$ residents. None of the interventions outlined in the letters were challenged by the families. Advocacy was needed for $16(4.5 \%)$ residents.

The time taken to administer medicines was collected pre- and postmedication review at eight similarly sized care homes. On average, 56.7 minutes per day or 6.6 hours per week of nursing was redirected from medicines administration to resident care.

Interviews with residents, families, care home staff and professionals were carried out. The main themes from interviews with residents and families were perceptions of over-prescribing, lack of understanding of the review process, and having no involvement in decisions about medicines. Medication reviews and the opportunity to be involved were highlighted as being valued by families. Care home staff noted medication administration rounds and lack of regular structured review as big concerns. The main benefits from the medication reviews were seen to be resident involvement and reduction in medicines burden. GPs acknowledged that they do not routinely involve patients in decisions and time was cited as a barrier to detailed reviews. They felt the reviews improved relationships between themselves, patients and care homes and that involving patients was a positive move. GPs and care home staff valued the contribution of the pharmacist in the review process. All staff valued the role that patients could play in their own health.

Fifty three of the 422 residents (13\%) required POAS input as follows:

- Nine POAS referrals for new POAS patients $(2.1 \%$ of residents reviewed)

- Fourteen POAS referrals for existing POAS patients (3.3\% of residents)

- Thirty-two requests for POAS advice (7.6\% of residents).

In the 422 residents reviewed, costs added owing to changing and starting medicines were $£ 4,138$ per annum and costs saved owing to changing or stopping medicines were $£ 81,989$ per annum, resulting in a net saving of $£ 77,702$ or $£ 184$ for every resident 
reviewed.

Limited data on medicines returned for destruction from the pharmacy supplying four care homes showed a downwards trend in medicines waste. There are data for one care home suggesting that reduction in waste was sustained eight months post-review.

To perform a basic health economic evaluation, we used the total number of interventions made and number of interventions where medicines were stopped as a marker for quality. We also factored in the net savings from changing, stopping or starting medicines.

Model 2 data should be interpreted with caution as only 21 residents were reviewed using this model. The number of interventions and medicines stopped were lowest in model 3. Models 1 and 4 had similar number of interventions and medicines stopped. The savings against the medicines budget were greatest for model 1 ( 234 per resident per annum) and considerably lower for model 3 ( $£ 101$ per resident per annum). In terms of service delivery, the cheapest was model 4 with no GP involvement ( $£ 58$ per resident). Full GP involvement (model 1) had the highest cost (£92 per resident). Moving from model 4 to model 1 would result in an increase of 0.3 interventions per resident but would cost an additional £34 per resident, per annum. Model 3 resulted in the least savings and fewest interventions.

For every £1 spent on service delivery (pharmacist, GP, care home nurse, POAS), the greatest saving was using model 0 ( $£ 3.53$ per resident) and the least for model 3 ( $£ 1.30$ per resident).

See supplementary file: ds4206.docx - "Summary of quantitative and qualitative results and models of service delivery"

\section{Lessons and limitations}

The aims and objectives we set out to achieve were met. The project benefited from strong relationships between the clinical and support teams and key stakeholders. A targeted communications strategy ensured maximum exposure of the project. Having a wide steering group ensured that all relevant professionals and patients were represented.

Furthermore, the clinical pharmacists involved were experienced independent prescribers who were competent to make autonomous decisions. The POAS consultant and team were fully dedicated to the project and had built in capacity to manage the additional workload that the project generated. Care home nurses and general practices were supportive and allowed full access to medical records.

We maximised patient involvement by first assuming all residents had capacity for medicines decisions. Final decisions were subsequently made by families or advocates only where residents lacked capacity. In cases where residents' families did not wish to be involved, we wrote to them in lay language explaining the interventions. involvement in care home reviews. This led to inconsistency in availability of GPs to participate in the MDT discussion. To account for this, the team accepted that one approach could not be sustained across all the practices we were planning to work with. Through consultation with lead GPs and practice managers we identified four models of GP involvement. The differential analysis of the models demonstrated that GP involvement in the MDT resulted in the greatest number of interventions. This may have limited the full potential of interventions and savings that could have been achieved had a GP be present in every review. Despite this, our development of other effective models of working has made the service more sustainable.

We have successfully shown an economic benefit to structured medication reviews where residents are involved in decisions. We further believe that our process can be upscaled and used to improve care for care home residents across the United Kingdom. The process that we have developed is also applicable to elderly patients who are not in care homes (eg sheltered housing or in their own homes) who have similar problems with inappropriate prescribing and lack of involvement. The shared decision making methodology could potentially be adopted for all those working with patients, irrespective of care setting

Limitations of this project include the fact that the care homes chosen were not randomly selected. A number of the qualitative interviews were taken from residents of families who expressed interest in taking part. Hence there may be bias in their observations.

Any interventions, including those identified as potentially causing harm, were not independently agreed or categorised according to the likelihood of harm. The subjective nature of these decisions could have limited their accuracy against more robust methods used to identify potential harm.[3]

The assessment of each resident's capacity to make decisions about medicines was based on the opinions of the care home nurse and/or GP who were present during the review. This lack of formal assessment created potential inaccuracy in the documented status. To test this, the POAS team performed both formal and informal assessments on a selection of residents in a project care home. The results showed that the informal assessment was accurate for the vast majority of cases. Although this goes some way to improve confidence in the methodology used, it is unclear whether the same would be true for the remainder of residents within the project.

Where medicines were started, changed or stopped, we estimated annualised costs and savings. The project did not follow-up each intervention to ensure the change was sustained for the following 12 months or whether the resident was still alive. A 2011 study commissioned by BUPA highlighted that residents had only a $55 \%$ chance of surviving the first year after admission.[9] However, as the average length of stay from that study was 801 days, and the overall savings from stopped medicines would continue for the life of that resident, we deemed the use of annualised savings to be reasonable. 


\section{BMJ Quality Improvement Reports}

Drug costs were taken from the Drug TariffTM at the time of the review. There may be inaccuracies in annual prices calculated as they may change over the course of the year.

Data on medicines waste was limited with information for four care homes at two months post-review and only one care home at eight months post-review. Further work is needed to ensure the suggested reduction in waste is replicated and sustained across all care homes within the study.

\section{Conclusion}

In conclusion, a structured multidisciplinary review of medicines in a care home setting where residents and/or their families are fully involved in decisions can improve quality of prescribing and reduce healthcare costs.

\section{References}

1. Gallagher P, O'Mahony D. STOPP (Screening Tool of Older Persons' potentially inappropriate Prescriptions): application to acutely ill elderly patients and comparison with Beers' criteria. Age Ageing 2008;37(6):673-9.

2. Parsons $\mathrm{C}$, Johnston $\mathrm{S}$, Mathie $\mathrm{E}$. Potentially Inappropriate Prescribing in Older People with Dementia in Care Homes:

A Retrospective Analysis. Drugs and Aging 2012;29(2):143-55.

3. Barber ND, Alldred DP, Raynor DK, et al. Care homes' use of medicines study: prevalence, causes and potential harm of medication errors in care homes for older people. Qual Saf Health Care 2009;18(5):341-6.

4. Age UK and The Health Foundation. Making Care Safer. Improving medication safety for people in care homes: thoughts and experiences from carers and relatives. London: The Health Foundation, 2011. http://www.health.org.uk/publications/making-care-safer (last accessed 21st Septemer 2014).

5. Implementing shared decision making. Clinical teams' experiences of implementing shared decision making as part of the MAGIC programme: Health Foundation, 2013. http://www.health.org.uk/public/cms/75/76/313/4173/MAGIC \%20evaluation.pdf?realName=hrsgE6.pdf (last accessed 23rd September 2014)

6. Hughes CM, Goldie R. "I just take what I am given": adherence and resident involvement in decision making on medicines in nursing homes for older people: a qualitative survey. Drugs \& Aging 2009;26(6):505-17.

7. Lewis DK, Robinson J, Wilkinson E. Factors involved in deciding to start preventive treatment: qualitative study of clinicians' and lay people's attitudes. BMJ 2003;327(7419):841.

8. Earthy A. Inappropriate use of medicines in acute care for the elderly related to a focus on acute care, providers' passive attitudes about learning, and paternalistic decision making. Evidence-Based Nursing 2006;9(3):96-97.

9. Forder J, Fernandez J-L. Length of stay in care homes, Report commissioned by BUPA Care Services,PSSRU
Discussion Paper 2769. Canterbury: PSSRU, 2011.

\section{Declaration of interests}

Nothing to declare.

\section{Acknowledgements}

The authors would like to thank the general practitioners, care home nurses, and managers, and the local clinical commissioning groups for supporting this project.

The authors would also like to acknowledge the project steering group: Annie Laverty, David Campbell, Peter Derrington, Yvonne Storey, Joan Lowerson, Aileen Beatty, Jane Riddle, Sandra Gray, John Connelly. 